\title{
Avulsion d'une dent surnuméraire incluse par découpe puis repositionnement de l'épine nasale antérieure
}

\author{
R. Gossart (Montpellier), C. Favre de Thierrens (Montpellier), J.H. Torres (Montpellier), \\ M.A. Fauroux (Montpellier)
}

Les avulsions de dents incluses sont d'une pratique courante. Le plus fréquemment, le dégagement osseux n'a pas de conséquences. Dans certains cas, il peut poser problème, s'il concerne un futur site d'implantation ou des structures anatomiques spécifiques, notamment dans le prémaxillaire (Sukegawa et al. Case Rep Dent. 2015; 2015: 974169). Pour ces situations, la piézochirugie apparait comme une technique peu invasive puisque la découpe est plus fine et plus précise qu'avec les instruments rotatifs (Pavlíková et al. Int J Oral Maxillofac Surg. 2011 40:451-7). De plus, le fait de découper un volet puis de le repositionner en fin d'intervention constitue un moyen de préserver le volume osseux. Le cas rapporté est celui d'une avulsion d'un mesiodens par découpe puis repositionnement de l'épine nasale antérieure. Une jeune fille de 13 ans a été adressée par son orthodontiste pour avulsion d'un mesiodens avant prise en charge ODF. Un examen cone beam a précisé la position de cet odontoïde. D’une morphologie évoquant une incisive conoïde, la dent surnuméraire se présentait en position verticale ; sa couronne faisait saillie dans les fosses nasales, sous le vomer, et sa racine était en arrière de celles des incisives centrales. Lors d'une intervention menée sous anesthésie générale, l'ENA a été découpée par piézochirugie (incision en $V$ de part et d'autre du plan médian) et luxée vers le haut, sans décollement de la muqueuse nasale. L'odontoïde a été sectionné au collet. Sa couronne d'abord puis sa racine ont été avulsées. Enfin, l'ENA a été repositionnée et fixée par une vis d'ostosynthèse positionnée légèrement en biais. Les suites ont été bonnes. La vis d'ostéosynthèse a été déposée un an plus tard. L'avulsion de cette dent, profondément incluse, par les techniques conventionnelles utilisant des instruments rotatifs aurait entraîné un délabrement important. Compte tenu de la situation inversée de l'axe de la dent, il aurait été difficile de la retirer par un abord palatin. De plus, son axe vertical compliquait la séparation couronne-racine, qui n'aurait été possible que par un accès nasal. Le choix d'une technique conservatrice de l'os (découpe puis repositionnement) semblait judicieux dans la mesure où il autorisait une bonne exposition du site et le morcellement cervical de la dent, tout en épargnant le volume osseux. Le fait de ne pas avoir décollé la muqueuse palatine a probablement contribué à la trophicité et la cicatrisation de l'ENA repositionnée. Les opérateurs ont pris le soin de ne pas placer la vis d'ostéosynthèse dans la suture entre les deux parties droite et gauche de l'ENA, afin de ne pas risquer de séparer ces deux os. Ce cas illustre une technique minimalement invasive qui a permis l'avulsion d'une dent surnuméraire profondément incluse en position verticale inversée, sans entraîner de lésion des dents voisines ni de la muqueuse nasale, tout en préservant le volume osseux de l'ENA. 